\title{
Design of Polarization-Independent Coarse Wavelength Splitters Based on Ridge-Waveguide Directional Couplers
}

\author{
Chee-Wei Lee \\ Cavendish Laboratory, University of Cambridge, J. J. Thomson Avenue, Cambridge CB3 OHE, UK \\ Correspondence should be addressed to Chee-Wei Lee, cwl30@cam.ac.uk
}

Received 28 April 2011; Revised 25 May 2011; Accepted 30 May 2011

Academic Editor: Vittorio M. N. Passaro

Copyright () 2011 Chee-Wei Lee. This is an open access article distributed under the Creative Commons Attribution License, which permits unrestricted use, distribution, and reproduction in any medium, provided the original work is properly cited.

We present the first unique design of a polarization-independent dual-wavelength splitter for wavelengths around $1.3 \mu \mathrm{m}$ and $1.55 \mu \mathrm{m}$ that is potentially of great interest to passive optical network (PON) applications. The filter design is simple compared with the other architectures and is based on ridge-type lateral directional couplers that can be readily integrated with other planar waveguide devices. Two design examples, based on InP/InGaAsP and Si/SiGe waveguides, are given. This polarization-independent wavelength splitting is achieved by exploiting the polarization dependence of the waveguides to produce coupling lengths that are sensitive to polarization and wavelength. We show that, to split the wavelengths without splitting the polarizations, the coupling lengths must be sufficiently different for TE and TM and for the different wavelengths in order to give the correct required ratios between the TE and TM coupling lengths for the two wavelengths of interest. We also show that the same approach can be applied to the design of a polarization splitter. The crosstalk, optical bandwidth, and fabrication sensitivity for the wavelength filter are evaluated.

\section{Introduction}

Wavelength splitting (demultiplexing) and combining (multiplexing) are important functions in many optical applications. An important example is fiber-to-the-home (FTTH) application, where $1.3 \mu \mathrm{m}$ and $1.5 \mu \mathrm{m}$ wavelength regions may be used to carry data/voice and video, respectively [1]. Since the wavelengths are spaced far apart, a coarse WDM (CWDM) filter would be needed to separate or combine the wavelengths. A bidirectional and compact filter that can be integrated with transceivers is desirable, as it would facilitate the development of low-cost terminal units for the home. Therefore, the filter should ideally be based on integrated optics, be polarization independent, and have large optical bandwidth and low crosstalk. An integrated optic realization of the ubiquitous fused fiber coupler is the waveguide directional coupler (DC). A dual-wavelength splitter for 1.3 and $1.55 \mu \mathrm{m}$, based on such a directional coupler, has been demonstrated in $\mathrm{SiGe} / \mathrm{Si}$ waveguides, but it is relatively large in size and works only for one of the polarizations [2].

In this paper, we show theoretically that compact and polarization-independent dual-wavelength splitters based on a single lateral directional coupler are possible for both the InGaAsP/InP and SiGe/Si material systems. The design is based on strongly confined or high-index contrast ridge waveguides, as opposed to the conventional weaklyguided, low-index contrast, rib or buried waveguides used in most other designs. Paradoxically, the key to achieving polarization independence is to utilize the strong waveguide birefringence inherent in these high-index-contrast waveguides. In the next section, we will present the design principle and constraints, from which it will become clear why the use of ridge waveguide is essential. The design principle can also be applied to polarization splitter. Then in Section 3, the birefringent nature of the waveguides will be briefly described, in Section 4, the dependence of the DC characteristics on waveguide parameters will be discussed, and in Section 5, the design approach will be illustrated for both $\mathrm{InP} / \mathrm{InGaAsP}$ and $\mathrm{Si} / \mathrm{SiGe}$ material platforms. Finally, in Section 6, the device performance is discussed in terms of crosstalk, optical bandwidth, and sensitivity, which was not discussed in [3].

It should be noted that the requirements and design of this CWDM waveguide filter are very different from 
narrowband or dense WDM (DWDM) waveguide filters, which are relatively complex and found in various forms based on vertical asymmetric waveguide couplers [4, 5], arrayed waveguide gratings [6], cascaded Mach-Zehnder interferometers [7], and many more.

\section{Design Principle of Polarization- Independent Wavelength Splitters}

The lateral directional coupler (DC) is the simplest waveguide structure that allows power transfer between two waveguides. If the waveguides are lossless and symmetric, then the output powers in the bar and cross waveguides are given, respectively, by [8]

$$
P_{b}=P_{\text {in }} \cos ^{2}\left(\frac{\pi}{2} \cdot \frac{L}{L_{c}}\right), \quad P_{c}=P_{\text {in }} \sin ^{2}\left(\frac{\pi}{2} \cdot \frac{L}{L_{c}}\right),
$$

where $P_{\text {in }}$ is the input power, $L$ is the interaction length and $L_{c}$ is the coupling length, which is the distance required for a complete power transfer. The coupling length is given by

$$
L_{c}(\lambda, \varepsilon)=\frac{\pi}{\kappa}=\frac{\lambda}{2\left[n_{e}(\lambda, \varepsilon)-n_{o}(\lambda, \varepsilon)\right]} \equiv \frac{\lambda}{2 \Delta n(\lambda, \varepsilon)},
$$

where $\kappa$ is the coupling coefficient, and $n_{e}$ and $n_{o}$ are the effective indices of the even and odd supermodes, respectively. The coupling length is in general polarization $(\varepsilon)$ and wavelength $(\lambda)$ dependent.

The periodic nature of the output power (as a function of distance) and the dependence of the coupling length on wavelength and polarization provide the mechanism for the DC to serve as a CWDM filter. This is because any two wavelengths, $\lambda_{1}$ and $\lambda_{2}$, assuming $\lambda_{2}>\lambda_{1}$, can be separated at the bar and cross ports if the interaction length $L$ of the directional coupler satisfies the condition

$$
L=m L_{c}\left(\lambda_{1}\right)=(m+p) L_{c}\left(\lambda_{2}\right)
$$

where $m$ and $p$ are integers and $p$ is odd. Note that $L_{c}\left(\lambda_{1}\right)>$ $L_{c}\left(\lambda_{2}\right)$ because the coupling coefficient $(\kappa)$ between two waveguides always increases with increasing wavelength. According to (3), $L$ could be an even multiple of $L_{c}\left(\lambda_{1}\right)$ at $\lambda_{1}$ and an odd multiple of $L_{c}\left(\lambda_{2}\right)$ at $\lambda_{2}$, as illustrated in Figure $1(\mathrm{a})$ for $m=2$ and $p=1$. We shall call the integer ratio $L / L_{c}$ the order. Clearly both orders (at the two wavelengths) should be small, since the smaller the orders and the $L_{c}$, the shorter the device will be. More importantly, it can be shown, as in Section 6, that the smaller the order, the more robust the design will be. On the other hand, for both orders to be small, $L_{c}$ must be very sensitive to wavelength. For instance, if $m=p=1$, then $L_{c}\left(\lambda_{1}\right)=2 L_{c}\left(\lambda_{2}\right)$, that is, $L_{c}$ has to change by a factor of 2 in going from $\lambda_{1}$ to $\lambda_{2}$. Such behavior may be possible only for certain types of waveguide and only for $\lambda_{1}$ and $\lambda_{2}$ that are sufficiently far apart.

The design is further constrained by the need to make the wavelength splitter polarization independent, such that both the TE and TM polarizations of a given wavelength exit the same port together. This requirement is difficult to meet. In general, it is not possible to satisfy (3) and at

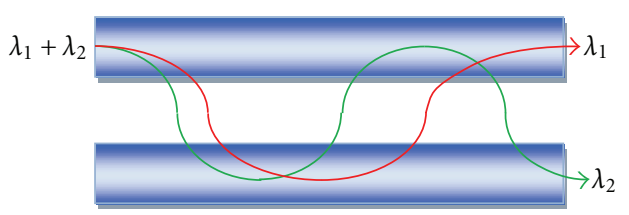

(a)

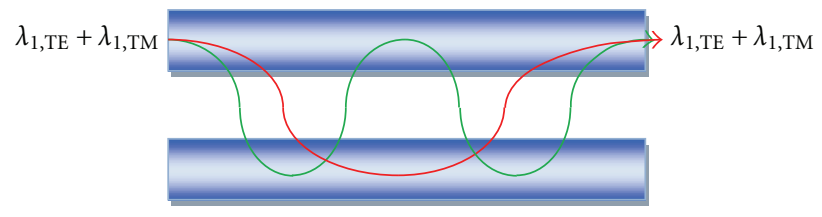

(b)

FIGURE 1: Illustration of input with (a) different wavelengths exiting at different ports (wavelength splitting) and (b) different polarizations for a single wavelength exiting at the same port.

the same time to have identical coupling length for both TE and TM. However, it is possible to be polarization independent if the coupling lengths for TE and for TM satisfy the condition: $L=m^{\prime} L_{c}^{\mathrm{TE}}(\lambda)=\left(m^{\prime}+q\right) L_{c}^{\mathrm{TM}}(\lambda)$ (where $q$ is an even integer), that is, the orders for TE and TM differ by an even integer, as illustrated in Figure 1(b). This condition assumes that the TE coupling length is larger than the TM, which we will see is true for directional couplers based on deep ridge waveguides. Altogether, for the directional coupler to split two wavelengths without splitting the polarizations, it must satisfy the multiple conditions:

$$
\begin{aligned}
L & =m L_{c}^{\mathrm{TE}}\left(\lambda_{1}\right)=(m+q) L_{c}^{\mathrm{TM}}\left(\lambda_{1}\right) \\
& =(m+p) L_{c}^{\mathrm{TE}}\left(\lambda_{2}\right)=\left(m+p+q^{\prime}\right) L_{c}^{\mathrm{TM}}\left(\lambda_{2}\right),
\end{aligned}
$$

where $p$ is odd and $q$ and $q^{\prime}$ are even. As an example that will be elaborated in Section 5, we consider the loworder combination $\left(m, p, q, q^{\prime}\right)=(1,3,2,4)$, which implies that $L_{c}^{\mathrm{TE}}\left(\lambda_{1}\right) / L_{c}^{\mathrm{TE}}\left(\lambda_{1}\right)=3, L_{c}^{\mathrm{TE}}\left(\lambda_{2}\right) / L_{c}^{\mathrm{TE}}\left(\lambda_{2}\right)=2$, and $L_{c}^{\mathrm{TE}}\left(\lambda_{1}\right) / L_{c}^{\mathrm{TE}}\left(\lambda_{2}\right)=4$. These relatively large ratios can be achieved only if the coupling lengths are strongly dependent on polarization and wavelength. This strong polarization and wavelength dependence is found only in directional coupler based on strongly guiding ridge waveguides in the singlemode regime. The properties of these ridge waveguides will be discussed in the next section. For other waveguides with small birefringence and wavelength dependence (such as the multimode interferometer [9]), the same method may be applied but the design might involve very high orders making the device very large. For this reason, we will only consider directional couplers based on single-mode ridge waveguides. For this type of wavelength filter, the general design procedure may be summarized as follows.

(1) For prespecified values of $\lambda_{1}$ and $\lambda_{2}$, we calculate the even and odd normal modes (for TE and TM), from which we obtain the coupling lengths, of the directional coupler at the two wavelengths.

(2) Repeat for various possible waveguide structures to identify the optimum zone. 
(3) From the various coupling length ratios obtained, determine the specific structure that yields a consistent set of $\left(m, p, q, p^{\prime}\right)$ that satisfies (4).

\section{High-Index-Contrast Ridge Waveguides}

Conventional optical waveguide devices are relatively large because the waveguides used are weakly guiding and do not allow routing of photons around sharp bends and corners. Such waveguides are therefore not suitable for even modest levels of device integration. In a drive towards high-density photonic integration, waveguides with strong lateral confinement, such as the ridge waveguide shown in Figure 2, have been developed in recent years because they permit sharper waveguide bends and smaller devices. Such waveguides also have different optical properties from conventional rib waveguides [3].

The lateral confinement in a ridge waveguide is strongly dependent on the waveguide width. Figure 3 (a) shows the effective indices $\left(n_{\text {eff }}\right)$ of all the guided optical modes as a function of the waveguide width, based on the InP/InGaAsP material system. The effective indexes are calculated for the optical wavelength of $1.55 \mu \mathrm{m}$ using a 3D finite-difference mode solver (Apollo APSS). Note that the width is less than about $0.8 \mu \mathrm{m}$ for a single-mode waveguide. In the singlemode region, the highly asymmetric mode profile gives rise to strong waveguide birefringence, with the TM effective index significantly larger than the TE. These effective indices change rapidly with the waveguide width. As the width is increased, the effective indexes approach each other and become equal at a critical width $\left(w_{c}\right)$. Above the critical width, the TE effective index becomes larger than of TM, as is the case for rib and slab-like waveguides. The rapid change of $n_{\text {eff }}$ with width in the single-mode regime also implies that the single-mode waveguides are the most sensitive to wavelength, as shown in Figure 3(b). These features will be utilized in our design of a polarization-insensitive CWDM filter.

\section{Structural Dependence}

We first consider directional couplers made of InP/InGaAsP ridge waveguides of width $w$ and separated by a gap size $g$. To maximize the wavelength and polarization dependence, $w$ should be in the single-mode regime (which is appropriate, as the directional coupler is a single-mode device), and the gap between the waveguides should be as deep as possible. To minimize the coupling length, the gap should be reasonably small but not too small as to pose fabrication difficulty. The effective indices of the supermodes in this directional coupler are calculated using the finite-difference mode solver, taking into account the wavelength dependence of the core refractive index [10]. Figure $4(\mathrm{a})$ shows the effective indices of TE and TM polarizations for $w=0.4 \mu \mathrm{m}, g=0.1$, and $0.3 \mu \mathrm{m}$. From this data, the coupling lengths are calculated using (2), and displayed in Figure 4(b). The effective indexes of the odd and even modes are calculated for the optical wavelength of $1.55 \mu \mathrm{m}$ using a 3D finite-difference mode solver (Apollo APSS). From the figures, we see that the

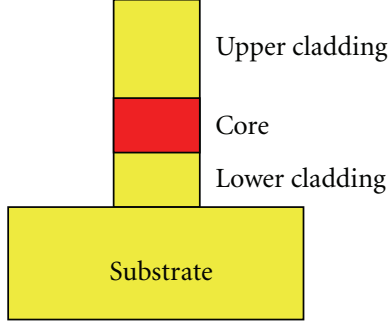

(a)

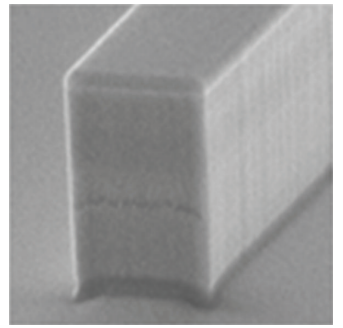

(b)
Figure 2: (a) Schematic and (b) SEM photo of the cross-section for a typical high-index-contrast ridge waveguide. High index contrast exists in the lateral direction at the core-air interfaces. The core could be InGaAsP or SiGe, and the claddings are $\mathrm{InP}$ or $\mathrm{Si}$, respectively.

coupling lengths (i) are fairly small (a few hundred $\mu \mathrm{m}$ ); (ii) are always larger for the TE polarization (this is true for deeply etched waveguides); (iii) decrease with increasing wavelength irrespective of the gap size; (iv) are more sensitive to wavelength for the larger gap size; note that from $\lambda_{1}=$ $1.31 \mu \mathrm{m}$ to $\lambda_{2}=1.54 \mu \mathrm{m}$, the TE coupling length changes by a factor of 4 for the case of $g=0.3 \mu \mathrm{m}$.

The ratio of the TE and TM coupling lengths are plotted in Figure 5 as a function of wavelength, and for two values of $w$. We see that the ratio $L_{c}^{\mathrm{TE}} / L_{c}^{\mathrm{TM}}$ can span a wide range of values; in particular, for $w=0.4 \mu \mathrm{m}$ and $g=0.3 \mu \mathrm{m}$, we note that $L_{c}^{\mathrm{TE}} / L_{c}^{\mathrm{TM}} \sim 3$ at $\lambda=1.31 \mu \mathrm{m}$ and 2 at $\lambda=1.54 \mu \mathrm{m}$, which match two of the ratios required. Furthermore, as mentioned earlier, $L_{c}^{\mathrm{TE}}(1.31 \mu \mathrm{m}) / L_{c}^{\mathrm{TE}}(1.54 \mu \mathrm{m}) \approx 4$ as can be seen from Figure 4(b). This structure, therefore, satisfies approximately all the requirements for a CWDM wavelength filter as specified by (4). By fine-tuning the waveguide parameters around these values, near perfect match to the requirements can be obtained, as will be shown in the next section.

\section{Design Examples}

As an exemplary study, Figure 6 shows the simulated power distribution along a $596 \mu \mathrm{m}$ long directional coupler, with $w$ $=0.395 \mu \mathrm{m}$ and $g=0.30 \mu \mathrm{m}$. With perfect order-matching, it can be seen that the two wavelengths are divided at the crossport and the bar port, respectively, with negligible crosstalk and polarization dependence. Simulation was performed with 3D beam propagation method using RSoft BeamPROP to verify the results obtained from (2) and the mode solver, and they are found to be similar. The simulations neglect any coupling at the input and output waveguides outside the interaction region, a good approximation since the total coupling here is found to be less than $2 \%$ if sharp bends with practical radius of $5 \mu \mathrm{m}$ are used to separate the access waveguides rapidly (this is possible because the waveguides are strongly confined). The coupling lengths obtained by simulation $\operatorname{are~} L_{c}^{\mathrm{TE}}(1.31 \mu \mathrm{m})=596 \mu \mathrm{m}, L_{c}^{\mathrm{TM}}(1.31 \mu \mathrm{m})=$ $199 \mu \mathrm{m}, L_{c}^{\mathrm{TE}}(1.54 \mu \mathrm{m})=149 \mu \mathrm{m}$, and $L_{c}^{\mathrm{TE}}(1.54 \mu \mathrm{m})=$ $75 \mu \mathrm{m}$. Hence, at $\lambda_{1}=1.31 \mu \mathrm{m}, L=L_{c}^{\mathrm{TE}}\left(\lambda_{1}\right)=3 L_{c}^{\mathrm{TM}}\left(\lambda_{1}\right)$, and at $\lambda_{2}=1.54 \mu \mathrm{m}, L=4 L_{c}^{\mathrm{TE}}\left(\lambda_{2}\right)=8 L_{c}^{\mathrm{TM}}\left(\lambda_{2}\right)$. These orders match the combination $(1,3,2,4)$ that satisfies (4). 


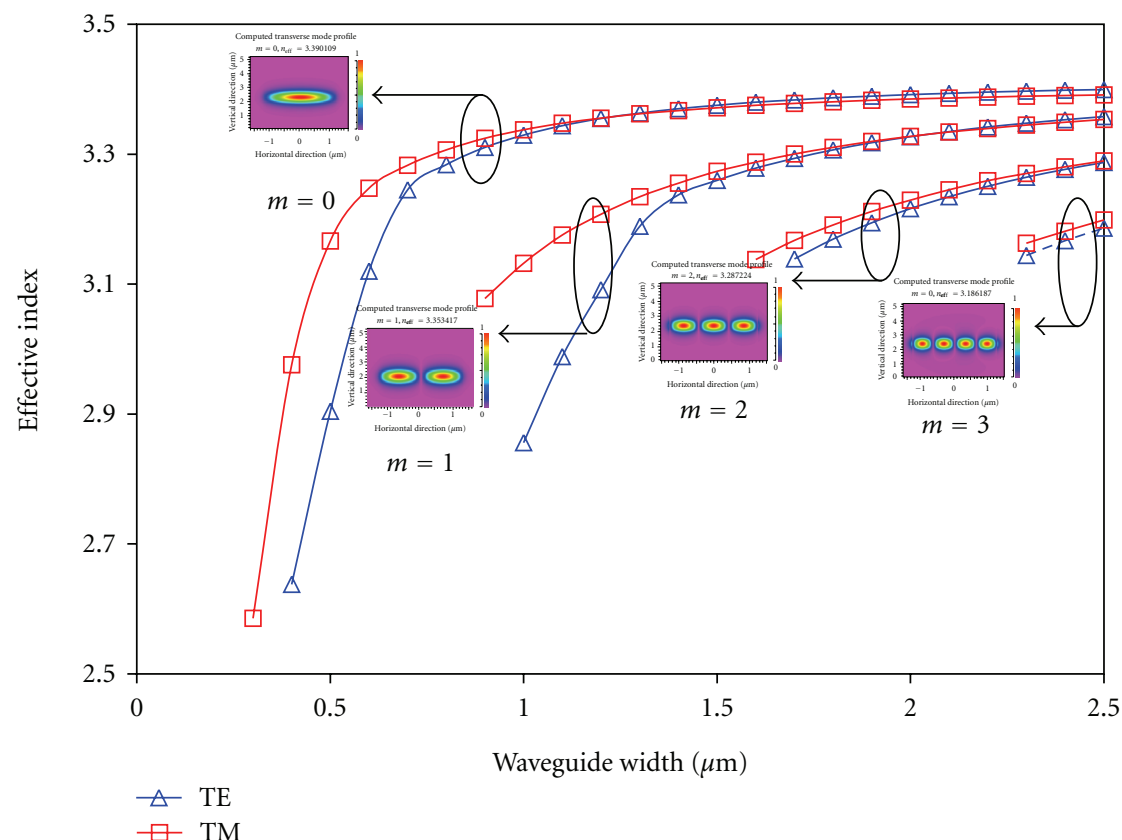

(a)

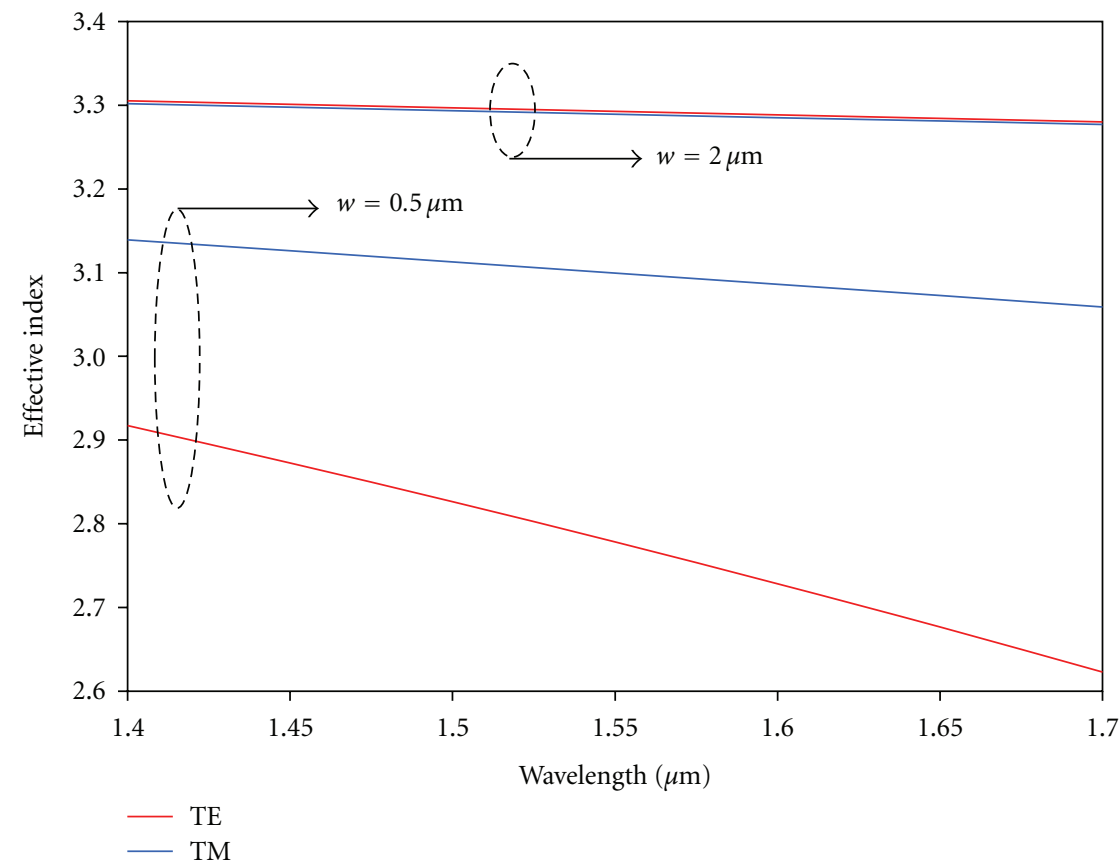

(b)

FIgUre 3: Effective indexes of InP/InGaAsP ridge waveguides for TE and TM polarizations as a function of (a) waveguide width and (b) operation wavelength. The InGaAsP core index is 3.5 while the InP cladding index is 3.17 . All core thicknesses are $0.65 \mu \mathrm{m}$.

We will further show that it is possible to apply the same approach to the Si-SiGe-based ridge waveguides. Silicon is attractive for passive photonic device applications due to its low cost and matured fabrication technology. For the SiGe core material, the material refractive index for TM is slightly larger than TE due to the lattice mismatch (strain) between SiGe and Si [11]. This material birefringence will reinforce the waveguide birefringence in the single-mode regime, resulting in a larger overall birefringence compared with the InP-based waveguides, and therefore, the coupling length ratios achievable are generally higher than the InP design. In our design example, we assume a waveguide with $\mathrm{Si}_{0.9} \mathrm{Ge}_{0.1}$ as the core material and $\mathrm{Si}$ as the cladding. This will give us the indexes summarized in Table 1 [11]. The core thickness is assumed to be $0.5 \mu \mathrm{m}$ in order not to exceed the critical thickness for strain relaxation [12]. 


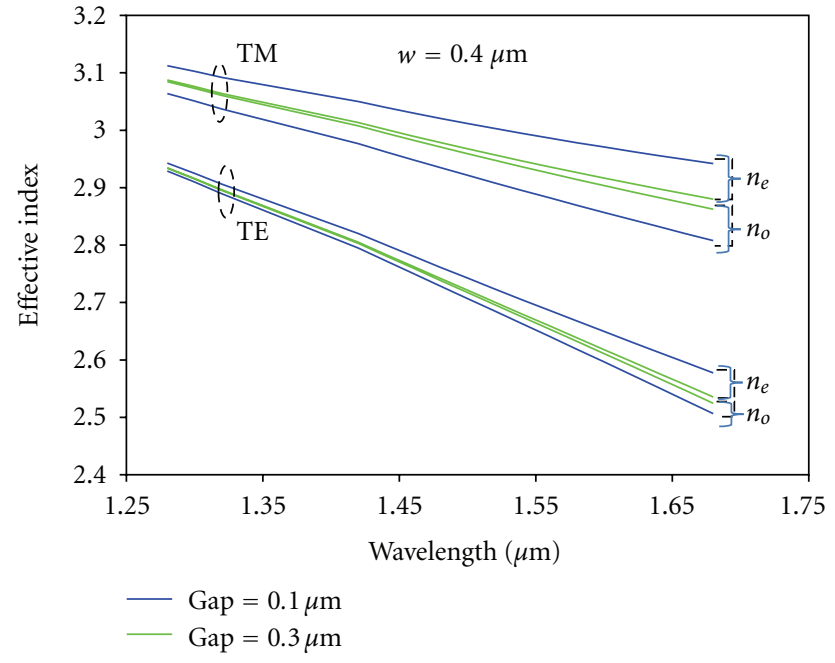

(a)

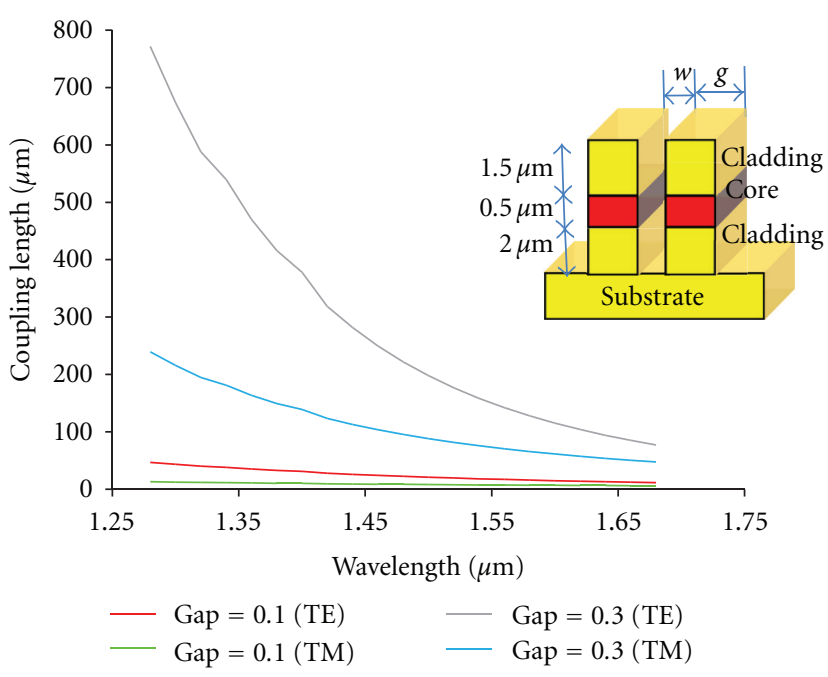

(b)

FIGURE 4: InP-based directional couplers: (a) effective indices (for even and odd supermodes) and (b) coupling lengths, for both TE and TM as a function of gap size $g$ (in $\mu \mathrm{m}$ ), with $w=0.4 \mu \mathrm{m}$. Inset shows a schematic of the directional coupler.

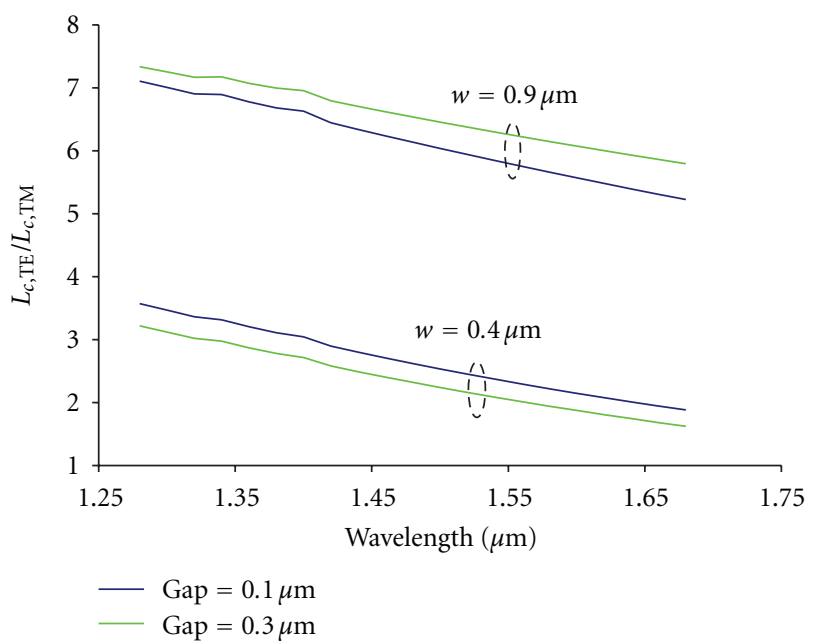

FIgure 5: The ratio between TE and TM coupling lengths as a function of wavelength, for various waveguide widths and gap sizes.

The DC wavelength filter is characterized by the combination $\left(m, p, q, q^{\prime}\right)=(1,3,4,12)$ (i.e., $L=L_{c}^{\mathrm{TE}}\left(\lambda_{1}\right)=5 L_{c}^{\mathrm{TM}}\left(\lambda_{1}\right)=$ $\left.4 L_{c}^{\mathrm{TE}}\left(\lambda_{2}\right)=16 L_{c}^{\mathrm{TM}}\left(\lambda_{2}\right)\right)$, which satisfies (4) for $\lambda_{1}=1.30 \mu \mathrm{m}$, $\lambda_{2}=1.56 \mu \mathrm{m}$. The waveguide parameters are $L=2829 \mu \mathrm{m}, w$ $=0.495 \mu \mathrm{m}$, and $g=0.30 \mu \mathrm{m}$. By contrast, the only reported case of a SiGe/Si DC dual-wavelength splitter [2] based on a rib waveguide structure has a length in excess of $5000 \mu \mathrm{m}$, but works only for the TE polarization (based on orders 4 and 5 , i.e., $\left.L=4 L_{c}^{\mathrm{TE}}\left(\lambda_{1}\right)=5 L_{c}^{\mathrm{TE}}\left(\lambda_{2}\right)\right)$.

Finally, we also note that our method based on matching different orders can be applied to the design of polarization splitter, which is another important component in integrated optics. This can be seen from the simulation result in Figure 6(b), where at $z=150 \mu \mathrm{m}$ the different polarizations of $\lambda=1.54 \mu \mathrm{m}$ have been separated to distinct ports.
TABLE 1: Refractive index of $\mathrm{Si}_{0.9} \mathrm{Ge}_{0.1}$ and $\mathrm{Si}$ materials for TE and TM modes of $\lambda=1.31 \mu \mathrm{m}$ and $1.55 \mu \mathrm{m}$.

\begin{tabular}{lcccc}
\hline \multirow{2}{*}{ Material } & \multicolumn{2}{c}{$\lambda=1.31 \mu \mathrm{m}$} & \multicolumn{2}{c}{$\lambda=1.55 \mu \mathrm{m}$} \\
& $\mathrm{TE}$ & $\mathrm{TM}$ & $\mathrm{TE}$ & $\mathrm{TM}$ \\
\hline $\mathrm{Si}$ & 3.508 & 3.508 & 3.476 & 3.476 \\
$\mathrm{Si}_{0.9} \mathrm{Ge}_{0.1}$ & 3.5395 & 3.5478 & 3.5065 & 3.5163 \\
\hline
\end{tabular}

Hence, the directional coupler terminated at this point would represent a compact polarization splitter for $\lambda=1.54 \mu \mathrm{m}$. In general, for a polarization splitter operating at a particular wavelength, the directional coupler is designed such that the orders of the coupling length for TE and TM polarizations differ by an odd integer at that wavelength. This design is extremely compact and straightforward compared to other guided wave-polarization splitters reported [13].

\section{Performance and Sensitivity}

6.1. Figure of Merit. The most important figure of merit for a wavelength filter is the wavelength isolation or crosstalk, which is defined as the ratio between the power at the unwanted wavelength $\left(\lambda_{u}\right)$ and the total power at the desired wavelength $\left(\lambda_{d}\right)$ at a specified output port, that is,

$$
\text { Crosstalk }(d B)=10 \log _{10}\left[\frac{P\left(\lambda_{u}\right)}{P_{\text {total }}\left(\lambda_{d}\right)}\right] \text {. }
$$

In (5), $P\left(\lambda_{u}\right)$ is the power for either TE or TM mode of the unwanted wavelength that is mixed at the output with the desired wavelength. It is given by either $P_{b}$ or $P_{c}$ in (1). Ideally, $P\left(\lambda_{u}\right)$ should be zero, giving zero crosstalk. However, crosstalk will increase when $\lambda, L$, or any other waveguide parameters deviate from their optimum values, since $P\left(\lambda_{u}\right)$ will increase according to $(1)$, and $L_{c}$ is a function of wavelength and all the waveguide parameters. 


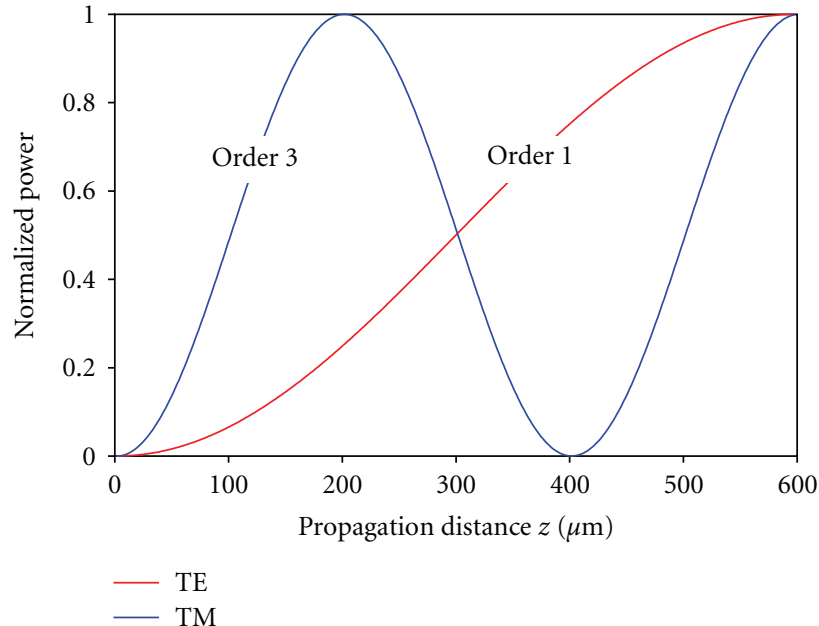

(a)

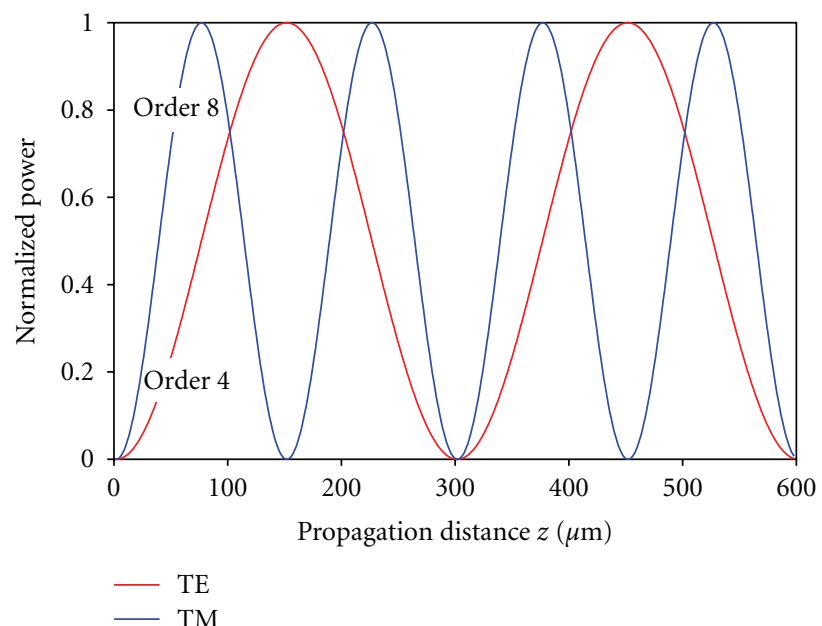

(b)

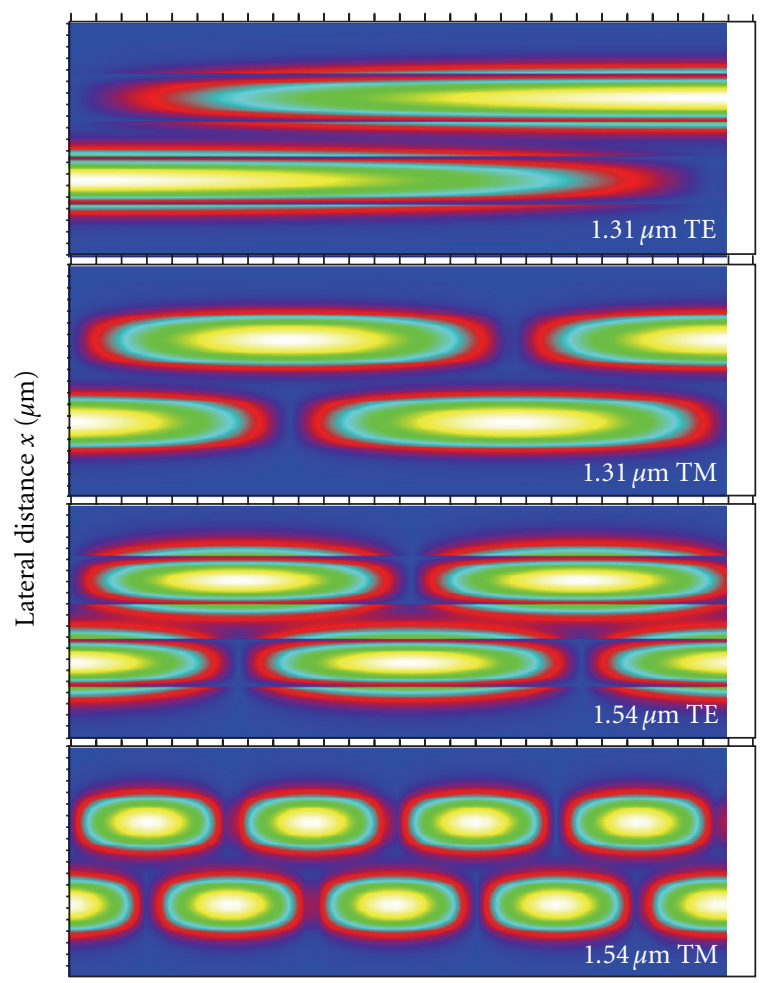

Propagation distance $z(\mu \mathrm{m})$

(c)

FIGURE 6: Simulated power in the cross waveguide for TE and TM polarizations of (a) $\lambda=1.31 \mu \mathrm{m}$, (b) $\lambda=1.54 \mu \mathrm{m}$, and (c) the corresponding field profiles along the directional coupler.

As an example, let us derive quantitatively the effect on the crosstalk at the bar port, where the desired output is $\lambda_{2}=$ $1.54 \mu \mathrm{m}$, due to a small deviation in $\lambda_{u}$ from the nominal value of $\lambda_{1}=1.31 \mu \mathrm{m}$. A small deviation in wavelength, $\Delta \lambda$, will cause a small deviation in coupling length, which may be represented by a dimensionless parameter $\delta$, such that

$$
1+\delta=\frac{L_{c}\left(\lambda_{1}\right)}{L_{c}(\lambda)}=\frac{\Delta n(\lambda)}{\Delta n\left(\lambda_{u}\right)} \cdot \frac{\lambda_{u}}{\lambda} \approx 1+\left.\frac{\Delta \lambda}{\Delta n\left(\lambda_{u}\right)} \cdot \frac{\partial \Delta n}{\partial \lambda}\right|_{\lambda_{u}}
$$

where we have used (2), and set $\lambda_{u} / \lambda \cong 1$. This gives the relationship between $\delta$ and $\Delta \lambda$, which can be written as

$$
\delta=\left.\frac{\Delta \lambda}{\Delta n\left(\lambda_{u}\right)} \cdot \frac{\partial \Delta n}{\partial \lambda}\right|_{\lambda_{u}}=\left.2 L_{c}\left(\lambda_{u}\right) \frac{\partial \Delta n}{\partial \lambda}\right|_{\lambda_{u}} \frac{\Delta \lambda}{\lambda_{u}} .
$$

Equation (1) can be used to give the power in the bar port at an arbitrary $\lambda$ that may be different from $\lambda_{1}$ :

$$
P_{b}(\lambda)=\cos ^{2}\left[M \frac{\pi}{2}(1+\delta)\right] \approx \sin ^{2}\left(M \frac{\pi}{2} \delta\right),
$$




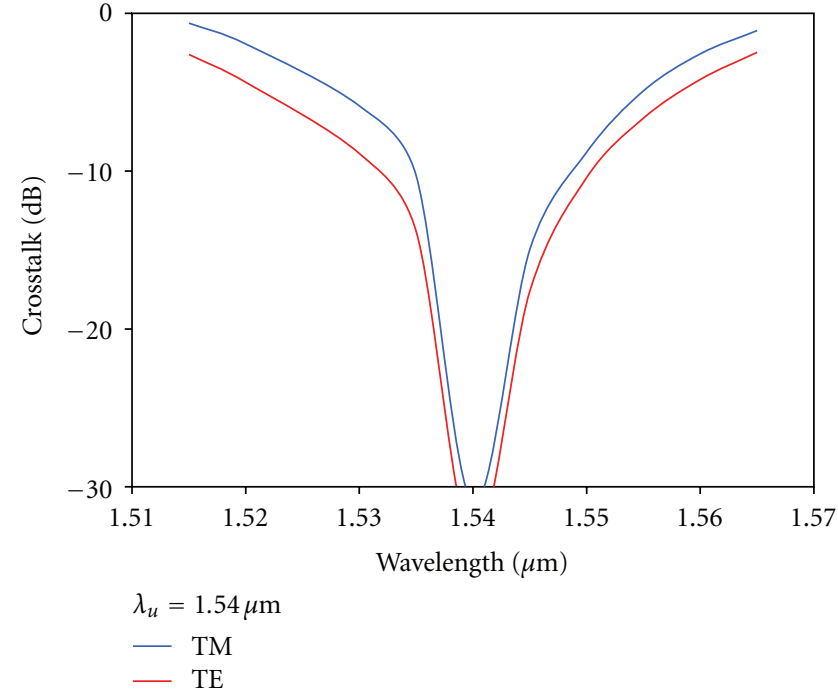

(a)

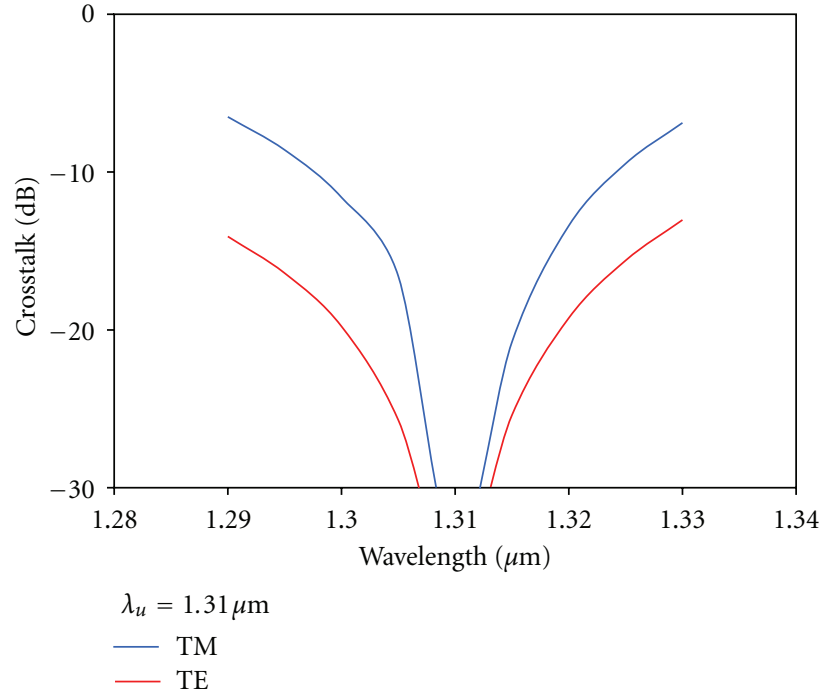

(b)

FIgure 7: The crosstalk for TE and TM polarizations at (a) $\lambda_{d}=1.31 \mu \mathrm{m}$ (at the cross-port) and (b) $\lambda_{d}=1.54 \mu \mathrm{m}$ (at the bar port).

where $M=L / L_{c}\left(\lambda_{1}\right)$ represents the order of coupling length at $\lambda_{1}$. Substituting (8) into (5), we obtain the crosstalk as

$$
\text { Crosstalk }(d B)=10 \log _{10}\left[\sin ^{2}\left(M \frac{\pi}{2} \delta\right)\right] \text {, }
$$

where we have assumed $P_{\text {total }}\left(\lambda_{d}\right)=1$. A typical acceptable crosstalk is $-20 \mathrm{~dB}$. To meet this requirement, the maximum $\delta$ that can be tolerated is

$$
\delta=\frac{0.02}{M \pi} . \quad(-20 \mathrm{~dB} \text { crosstalk })
$$

Hence, we can see that the smaller the order used, the larger will be the tolerable coupling length deviation $\delta$. Using (7), the wavelength tolerance $\Delta \lambda=|\lambda-\lambda u|$ is given by

$$
\frac{\Delta \lambda}{\lambda_{u}}=\frac{\delta}{\left.2 L_{c}\left(\lambda_{u}\right) \cdot \frac{\partial \Delta n}{\partial \lambda}\right|_{\lambda_{u}}}=\frac{0.01}{\left.\mathrm{M} \pi L_{c}\left(\lambda_{u}\right) \cdot \frac{\partial \Delta n}{\partial \lambda}\right|_{\lambda_{u}}} .
$$

Hence, to maximize $\Delta \lambda$, it is desirable to make $M$ and $L_{c}$ as small as possible. $\Delta \lambda$ represents the optical bandwidth for a given crosstalk, that is, the range of wavelengths for which the specified crosstalk is satisfied. It is an important measure of the wavelength sensitivity of the filter. For the CWDM application considered here, the optical bandwidth should be as large as possible so that no temperature stabilization of the laser wavelength is needed.

The same analysis can be applied to the cross-port to either wavelengths, and to either TE or TM. In addition to $\lambda$, the coupling length is also dependent on the waveguide structure, namely the gap size and the waveguide width. Similar analysis can be used to derive the degradations in crosstalk due to variations in these dimensions, thereby giving the sensitivity of the device performance to these parameters.
So far, we have not considered the propagation loss in the waveguides. An important parameter is the polarizationdependent loss (PDL), which is not insignificant for singlemode ridge waveguides. The PDL is very sensitive to the waveguide width. For the InP waveguide with $w=0.4 \mu \mathrm{m}$, previously reported results at $\lambda=1.54 \mu \mathrm{m}$ are about $9 \mathrm{~dB} / \mathrm{cm}$ and $7 \mathrm{~dB} / \mathrm{cm}$ for TE and TM, respectively [3]. The PDL is not excessive if the device is sufficiently short, which is another reason to keep $L$ small. For the design example above where $L \sim 600 \mu \mathrm{m}$, the PDL is about $0.1 \mathrm{~dB}$. However, PDL should be included when considering the crosstalk for different polarizations.

6.2. Sensitivity. For the InP example discussed above, the crosstalk at the cross-port $\left(\lambda_{d}=1.31 \mu \mathrm{m}\right)$ and the bar port $\left(\lambda_{d}=1.54 \mu \mathrm{m}\right)$ as a function of wavelength deviation for different polarizations are given in Figure 7. The polarizationdependent loss mentioned above has been included in calculating the crosstalk for different polarizations. Note that the crosstalk and the optical bandwidth (for a specified crosstalk) are different for different polarizations and for different wavelengths (ports). In the case of $\lambda_{d}=1.3 \mu \mathrm{m}$, the bandwidth is about $20 \mathrm{~nm}$ for TE and $10 \mathrm{~nm}$ for TM. In the case of $1.54 \mu \mathrm{m}$, the optical bandwidths are 7 and $5 \mathrm{~nm}$ for TE and TM, respectively. These results reflect the order dependence of (11): the lower the order, the larger the bandwidth.

Similarly, Figure 8 shows the effects of dimensional variations in the waveguide width and the gap separation on crosstalk for the InP design example. It can be seen that, to meet a typical crosstalk requirement of $-20 \mathrm{~dB}$, the waveguide width must be controlled to within $\pm 5 \mathrm{~nm}$ and the gap size to within $\pm 4 \mathrm{~nm}$. These requirements will in general depend also on the order of the output functions. In the $\mathrm{Si} / \mathrm{SiGe}$ case, for example, the sensitivity will be worse because of the higher orders involved. 


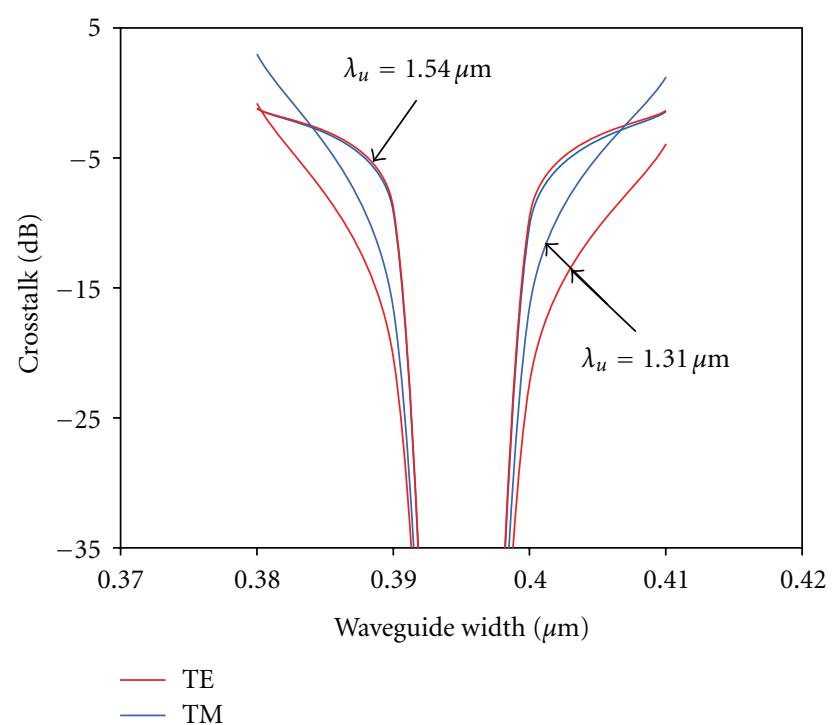

(a)

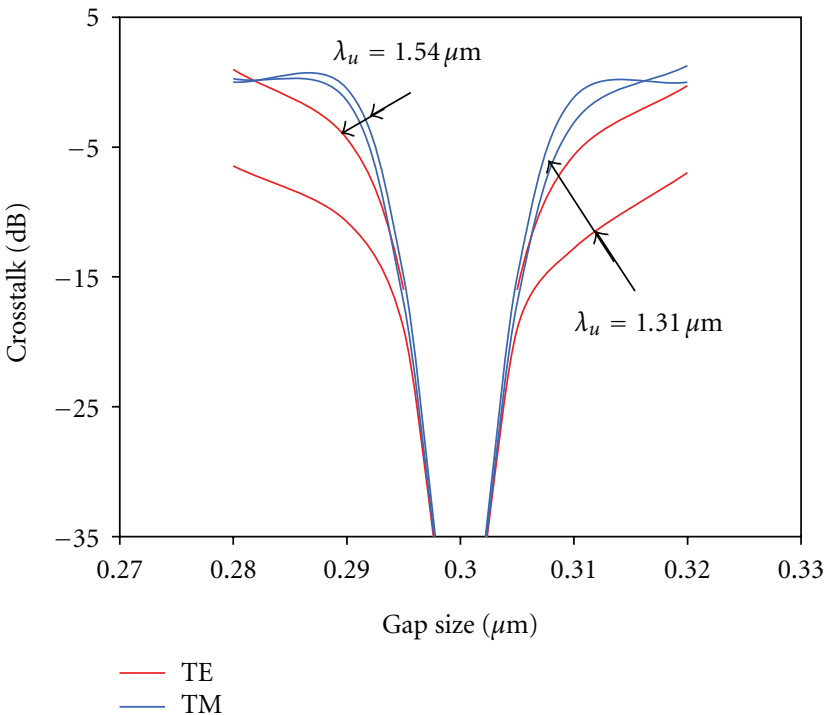

(b)

FIGURE 8: TE and TM crosstalks at the bar port and the cross-port of the CWDM directional coupler for various (a) waveguide widths and (b) gap sizes. All dimensions are in $\mu \mathrm{m}$.

\section{Conclusion}

We have shown, theoretically, that it is possible to design directional couplers that can split two wavelengths spaced very far apart in a polarization-insensitive manner. We have considered the wavelength components around $1.3 \mu \mathrm{m}$ and $1.5 \mu \mathrm{m}$ regions, where this type of CWDM filter is potentially of great interest to passive optical networks (PON) technology. Although the design principle, based on matching the orders of coupling length, is well known, no feasible theoretical and practical designs have been demonstrated so far, primarily because they have been based on conventional weakly guiding waveguides. In this paper, we show for the first time theoretical designs for polarization-independent dual-wavelength splitters, for both InP- and Si-based materials. The key to the design is to exploit the highly polarization-dependent feature of strongly confined ridge waveguides. Only these waveguides make possible directional couplers with coupling lengths that span over the large range required to achieve polarizationindependent wavelength splitting. The particular design for InP discussed above occurs in the single-mode regime with strong birefringence, where the birefringence (and coupling length) is very sensitive to the wavelength, as well as the waveguide width and other structural parameters. Hence, the fabrication requirement is very stringent, but may be achieved with advanced nanofabrication technology. Finally, we have also shown that our design approach can be generalized to the design of very compact polarization mode splitters.

\section{Acknowledgments}

The author would also like to express his sincere appreciation to the guidance of the late Associate Professor Mee-Koy
Chin. The author also gratefully acknowledge the support of $A^{*}$ STAR Singapore through the postgraduate and the overseas postdoctoral sponsorships.

\section{References}

[1] H. Kawata, T. Ogawa, N. Yoshimoto, and T. Sugie, "Multichannel video and IP signal multiplexing system using CWDM technology," Journal of Lightwave Technology, vol. 22, no. 6, pp. 1454-1461, 2004.

[2] M. R. T. Pearson, P. E. Jessop, D. M. Bruce, J. Ojha, and S. Kovacic, "SiGe-based dual-wavelength demultiplexer and polarization splitters," in Silicon-Based Optoelectronics, vol. 3630 of Proceedings of SPIE, pp. 29-39, San Jose, Calif, USA, January 1999.

[3] M.-K. Chin, C.-W. Lee, S.-Y. Lee, and S. Darmawan, "Highindex-contrast waveguides and devices," Applied Optics, vol. 44, no. 15, pp. 3077-3086, 2005.

[4] B. Liu, A. H. Shakouri, P. Abraham, Y. J. Chiu, S. Zhang, and J. E. Bowers, "Fused InP-GaAs vertical coupler filters," IEEE Photonics Technology Letters, vol. 11, no. 1, pp. 93-95, 1999.

[5] W. Chi, C. Rolland, F. Shepherd et al., "InGaAsP/InP vertical directional coupler filter with optimally designed wavelength tunability," IEEE Photonics Technology Letters, vol. 5, no. 4, pp. 457-459, 1993.

[6] C. R. Doerr, R. Pafchek, and L. W. Stulz, "Integrated band demultiplexer using waveguide grating routers," IEEE Photonics Technology Letters, vol. 15, no. 8, pp. 1088-1090, 2003.

[7] B. J. Offrein, G. L. Bona, F. Horst, H. W. M. Salemink, R. Beyeler, and R. Germann, "Wavelength tunable optical addafter-drop filter with flat passband for WDM networks," IEEE Photonics Technology Letters, vol. 11, no. 2, pp. 239-241, 1999.

[8] R. A. Forber and E. Marom, "Symmetric directional coupler switches," IEEE Journal of Quantum Electronics, vol. 22, no. 6, pp. 911-919, 1986.

[9] L. B. Soldano and E. C. M. Pennings, "Optical multi-mode interference devices based on self-imaging: principles and 
applications," Journal of Lightwave Technology, vol. 13, no. 4, pp. 615-627, 1995.

[10] B. Broberg and S. Lindgren, "Refractive index of $\mathrm{In}_{1-x} \mathrm{Ga}_{x} \mathrm{As}_{y} \mathrm{P}_{1-y}$ layers and $\mathrm{InP}$ in the transparent wavelength region," Journal of Applied Physics, vol. 55, no. 9, pp. 3376 3381, 1984.

[11] S. Janz, J.-M. Baribeau, A. Delâge et al., "Optical properties of pseudomorphic $\mathrm{Si}_{1-x} \mathrm{Ge}_{x}$ for Si-based waveguides at the $\lambda=1300-\mathrm{nm}$ and 1500-nm telecommunications wavelength bands," IEEE Journal on Selected Topics in Quantum Electronics, vol. 4, no. 6, pp. 990-995, 1998.

[12] R. People, "Physics and applications of $\mathrm{Ge}_{x} \mathrm{Si}_{1-x} / \mathrm{Si}$ strainedlayer heterostructures," IEEE Journal of Selected Topics in Quantum Electronics, vol. 22, no. 9, pp. 1696-1710, 1986.

[13] J. J. G. M. van der Tol, J. W. Pedersen, E. G. Metaal, J. J.-W. van Gaalen, Y. S. Oei, and F. H. Groen, "A short polarization splitter without metal overlays on InGaAsP-InP," IEEE Photonics Technology Letters, vol. 9, no. 2, pp. 209-211, 1997. 

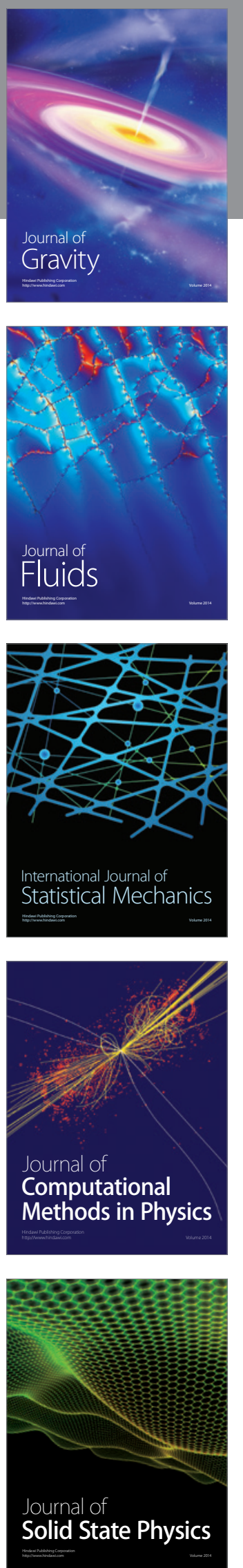

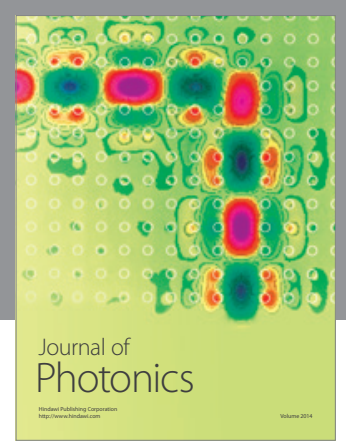

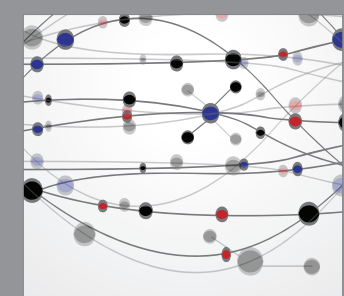

The Scientific World Journal
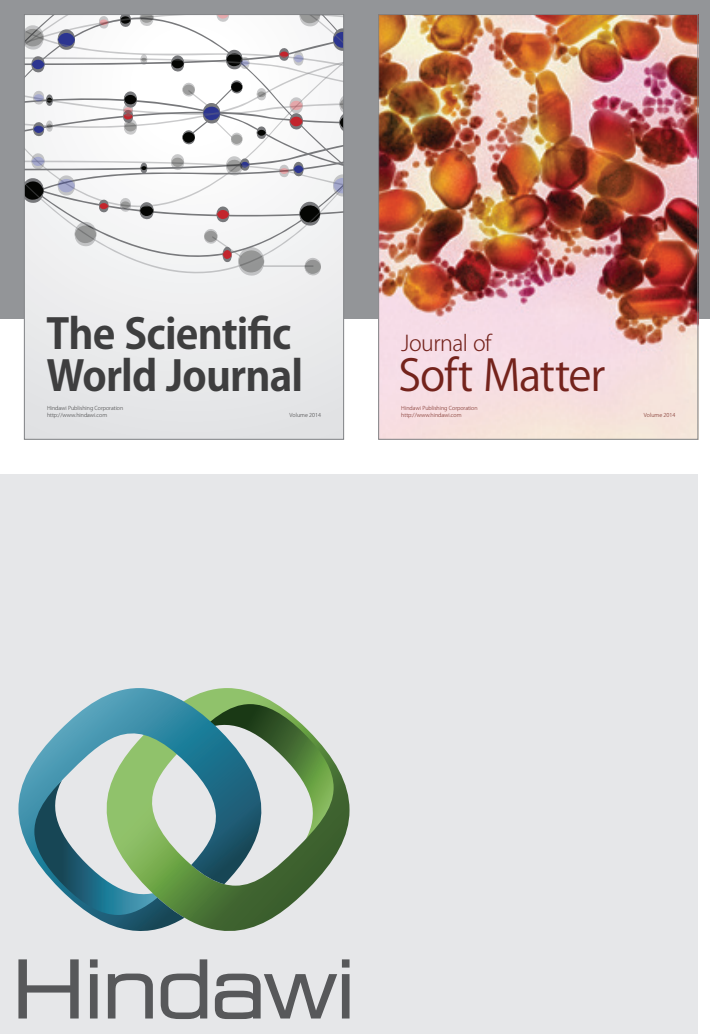

Submit your manuscripts at

http://www.hindawi.com
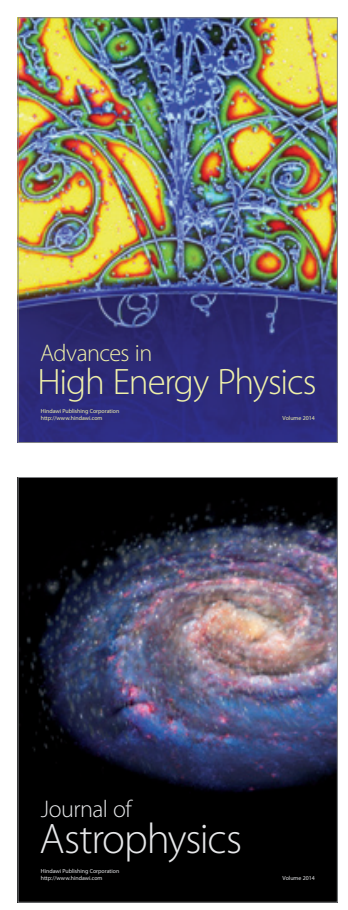
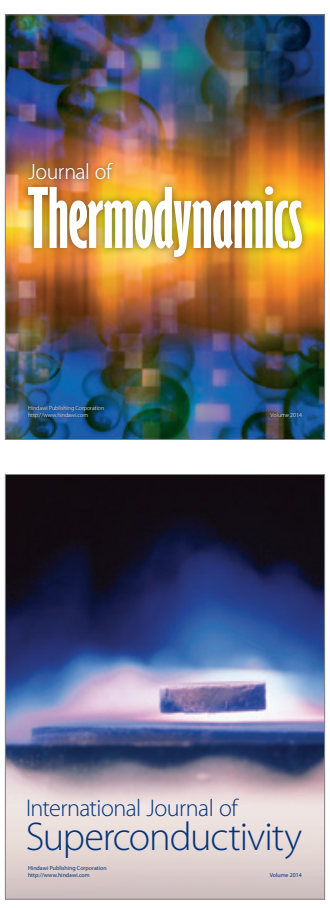
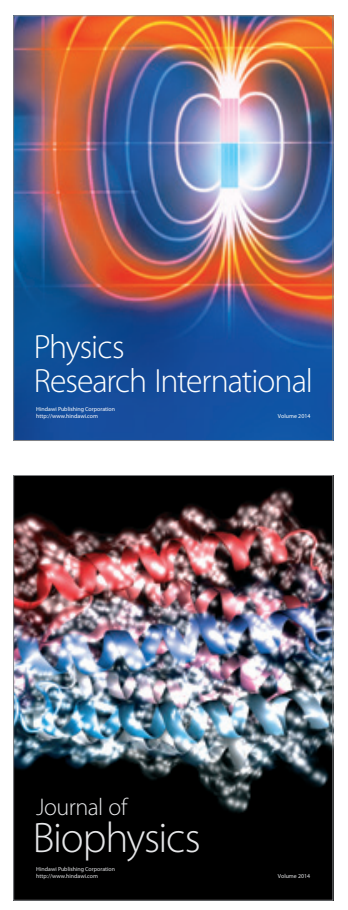
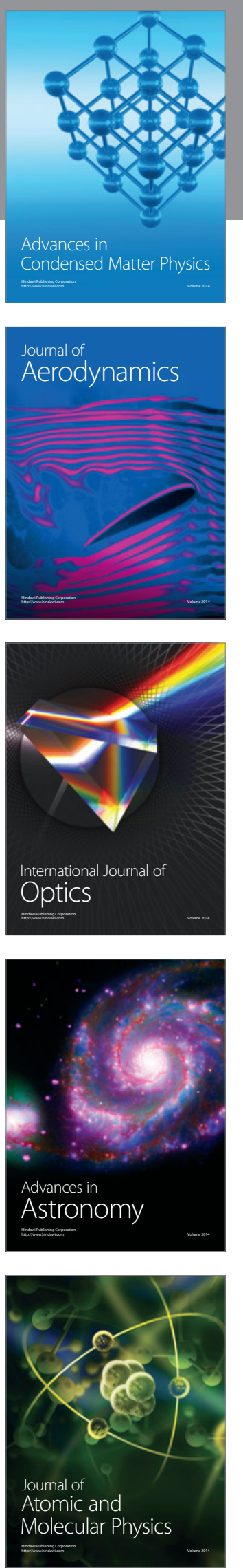